\title{
TV/Series
}

12 | 2017

Littérature et séries télévisées/Literature and TV series

\section{"This is not my hat », les accessoires de Sherlock, du canon à la série $\mathrm{BBC}$}

\section{Hélène Machinal}

\section{(2) OpenEdition}

\section{Journals}

Édition électronique

URL : http://journals.openedition.org/tvseries/2187

DOI : 10.4000/tvseries.2187

ISSN : 2266-0909

Éditeur

GRIC - Groupe de recherche Identités et Cultures

Référence électronique

Hélène Machinal, « "This is not my hat », les accessoires de Sherlock, du canon à la série BBC », TV/ Series [En ligne], 12 | 2017, mis en ligne le 20 septembre 2017, consulté le 21 avril 2019. URL : http:// journals.openedition.org/tvseries/2187; DOI : 10.4000/tvseries.2187

Ce document a été généré automatiquement le 21 avril 2019

\section{(c) $(1)$}

TV/Series est mis à disposition selon les termes de la licence Creative Commons Attribution - Pas d'Utilisation Commerciale - Pas de Modification 4.0 International. 


\title{
« This is not my hat ", les accessoires de Sherlock, du canon à la série $\mathrm{BBC}$
}

\author{
Hélène Machinal
}

1 Travailler sur la question de l'adaptation du personnage de Holmes et du canon Doylien par Moffat et Gatiss dans la série Sherlock (BBC, 2010-) est un vrai bonheur. On y retrouve en effet toutes les lignes de force du texte source, Holmes, Watson, Mycroft, Mrs Hudson, Lestrade, Moriarty, Adler, Londres, le 221B, mais aussi des références aux nouvelles les plus connues: A Study in Scarlet, The Sign of Four, The Hound of the Baskervilles, "A Scandal in Bohemia »... La différence majeure et évidente entre source textuelle et série est bien entendu la transposition du contexte et le passage d'une episteme post-darwiniene à une episteme post-cybernétique. Les correspondances entre les deux contextes socio-culturels sont cependant flagrantes: à l'essor de la presse périodique et du roman feuilleton correspond cette autre forme de fiction "populaire " que sont les séries $\mathrm{TV}^{1}$. Le succès populaire du personnage est lié à l'explosion d'un marché de diffusion et à la sérialisation, à un fascination très spécifique d'un public vite amené à assimiler fiction et réalité, à un cristallisation de la figure du détective, et surtout, à un phénomène d'autonomisation de la fiction par le biais duquel l'auctorialité disparaît au profit de la survie exclusive de la création. Toutes ces caractéristiques témoignent de la proximité entre le phénomène culturel et économique que put représenter le roman feuilleton à la fin du XIx et celui des séries TV et de la culture sérielle au tournant du xxe et du xxI siècle.

\section{Vous avez dit « adaptation »?}

2 L'incipit du premier épisode de la série Sherlock de la BBC nous permet d'emblée de caractériser le type d'adaptation à l'œuvre ${ }^{2}$. Il nous projette sans crier gare dans des scènes de type documentaire caractérisées par le port de la caméra à l'épaule et une prise de vue subjective. 
Figure 1 : BBC Sherlock, 1.1
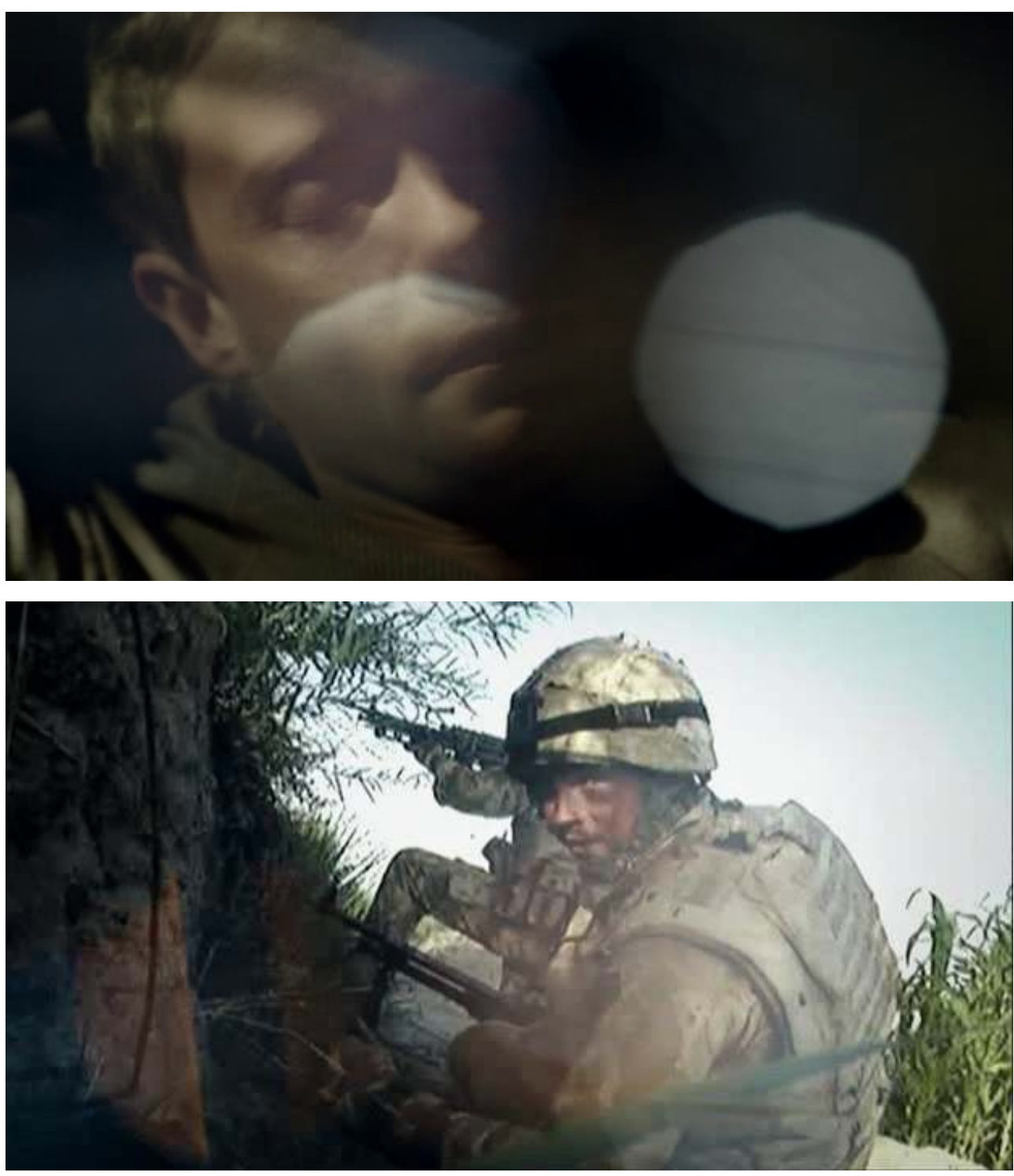

L'authenticité documentaire est ainsi garantie. Les scènes qui suivent révèlent, par montage alterné entre dormeur agité et scène de combat, qu'il s'agit d'un cauchemar où le réalisme documentaire nie implicitement la nature irréelle du rêve. En l'espace d'une minute 33, l'incipit nous présente donc un conflit, un ancien soldat qui boite, son blogqui révèle aussi son nom - et la psychiatre qui le suit. Cet incipit renvoie aussi immédiatement le lecteur de Doyle aux premières pages de A Study in Scarlet, où Watson décrit son retour d'Afghanistan et son arrivée à Londres suite à une blessure à l'épaule, puis sa rencontre avec Stamford qui va conduire à la co-location du 221b Baker Street ${ }^{3}$. D'une guerre d'Afghanistan à l'autre, la transposition à l'œuvre est bien entendu avant tout contextuelle et temporelle. On retiendra le blog de Watson qui bien que présenté pour ses vertus thérapeutiques, introduit immédiatement la dimension narrative et la sémiotique propre à la lecture.

Les trois critères de l'adaptation avancés par Hutcheon sont ainsi omniprésents dès le départ: transposition d'une fin de siècle à l'autre, intertextualité soulignée avec les nouvelles de Doyle, mais aussi appropriation par une propension à la création qui s'inscrit 
précisément, pour reprendre les termes de Marie-Claire Ropars-Wuilleumier ${ }^{4}$ dans ces « lacunes » ou « inquiétudes de soi » que l'adaptation tend à rendre visible.

5 L'intertextualité se décline sur plusieurs modes et ne se dément pas au fil de la production des divers épisodes. Les citations sont rarement directes et Christophe Gelly a déjà souligné la distance ironique des références à l'hypotexte ${ }^{5}$.

Figure 2 : Effets de fiction, 2.1

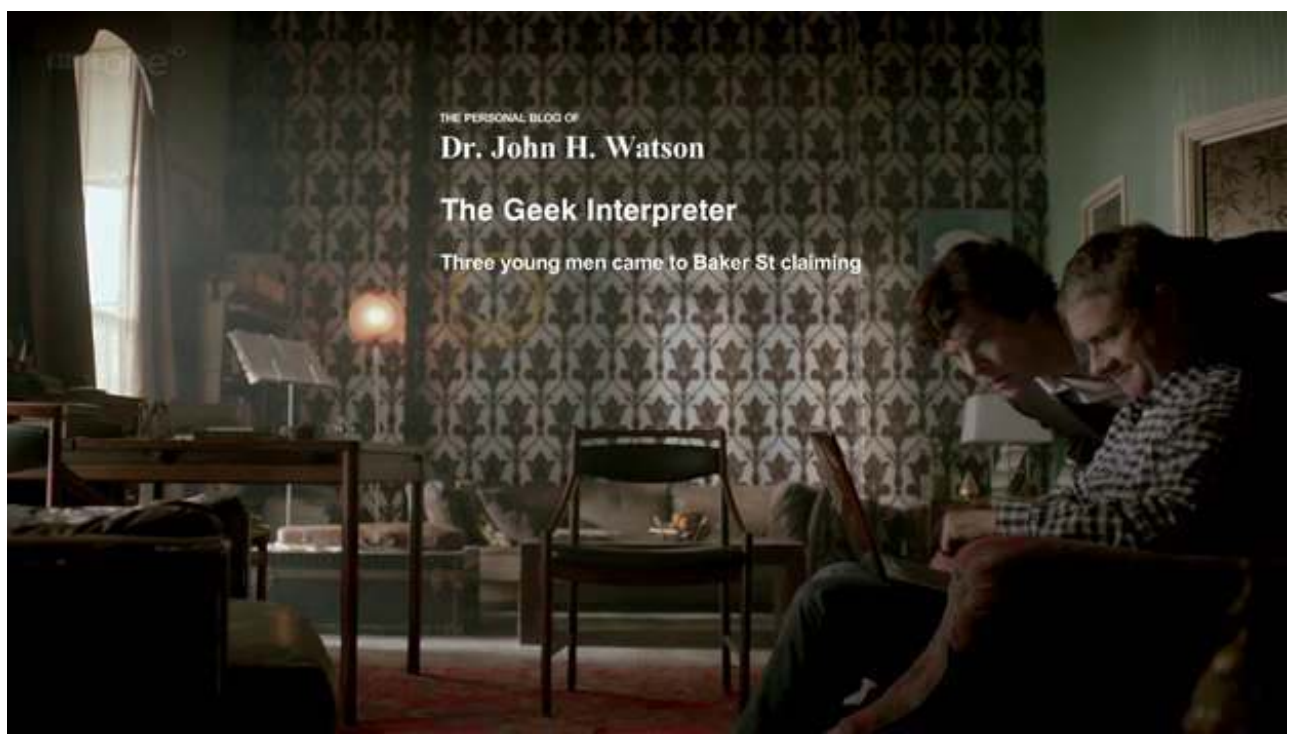

6 Ainsi des renvois aux titres des nouvelles de Doyle légèrement modifiés par un jeu homophonique qui apparaît dès le titre du premier épisode : "A Study in Pink». Plus subtilement, le jeu avec l'hypotexte prend un tour particulier lorsqu'au début de « $\mathrm{A}$ Scandal in Belgravia", nous rencontrons un écho à une pratique, cette fois métaréférentielle, de Doyle qui consiste à faire allusion en début de nouvelle à des titres d'enquêtes que sont censés avoir menés les deux personnages mais qui ne figurent pas dans les récits publiés dans le Strand Magazine ${ }^{6}$. Moffat et Gatiss prouvent d'emblée leur inventivité et leur capacité à la transposition puisque ces allusions hypotextuelles renvoient aussi dans la série de la BBC à un « effet de fiction », c'est-à-dire à des nouvelles qui n'ont pas été mises en scène ou produites mais néanmoins écrites par Watson ... sur son blog. "The Speckled Band» devient "The Speckled Blond» ou "The Greek Interpreter » devient « The Geek Interpreter » mais la mise en abyme de la fiction par la fiction s'opère au détriment de l'illusion de réalité dans l'original car ces nouvelles existent bien «narrativement » dans le canon, elles font même partie des nouvelles les plus connues et elles sont ici renvoyées au statut d'effet de fiction qu'avaient les nouvelles que mentionne Watson sans jamais les écrire. Or, gardons en mémoire que ces nouvelles « fictives » dans le canon ont été la matrice de nombreux pastiches et autres réécritures qui contribuèrent à l'autonomie de la figure mythique et à sa plasticité.

7 Le jeu métaréférentiel opère donc à plusieurs niveaux d'interaction entre fiction et réalité et se joue des pratiques qui étaient déjà présentes dans l'original. Ce jeu s'adresse en premier lieu au spectateur que la série maintient en contact constant avec la référence tout en s'appliquant à divers types de décalages : décalage citationnel comme dans « The game is on " et non plus " The game is afoot ${ }^{7}$ ", condensation citationnelle comme lorsque Watson traite Holmes de "machine ${ }^{8}$ ", ou expansion citationnelle lorsque dans "A Scandal in Belgravia », Holmes et Adler sont présentés l'un à l'autre in abstentia via des 
clichés photographiques alors que dans la nouvelle «A Scandal in Bohemia », tout ce que Holmes gardera de Irène Adler est une unique photo. Avec Moffat et Gatiss, son unique memento est son " camera phone ».

Ce repérage succinct montre à loisir qu'adaptation est réellement synonyme de création dans cette nouvelle version de Sherlock. Cependant, ce qui me semble plus intéressant dans la série est une pratique de la distanciation par la référence qui contribue à mettre en lumière ces «inquiétudes de soi » qui vont s'avérer être à la fois à l'origine de l'accession du détective au statut de figure mythique mais aussi permettre un réel travail sur le transfert problématique d'une sémiotique de la lecture à une sémiotique de l'image.

\section{«I prefer to do my own editing » $(2,2.6$ :30)}

L'opposition entre deux régimes sémiotiques distincts et spécifiques est souvent l'argument avancé à la fois pour déprécier l'image en tant que médium, mais aussi pour la réduire et la cantonner à une position subalterne où l'adaptation se pense en termes de pur modèle et pâle réplique. Il reste que le récit policier présente un cas d'étude particulièrement pertinent puisque le processus narratif fonde la dynamique du récit. On se souvient en effet du rôle crucial du narrateur qui, dans ce récit impossible qu'est le récit policier, doit à la fois ménager l'intérêt du lecteur en lui donnant l'illusion qu'il pourra parvenir à la solution avant le détective, et garder sa fonction première qui consiste à ériger le détective en figure de la toute-puissance sémiotique ${ }^{9}$.

La question de la transposition en images de cette construction narrative très particulière est d'autant plus intéressante qu'elle revient à poser la différence entre ces deux régimes sémiotiques spécifiques. Comment s'affranchir du modèle de la lecture alors que le détective est avant tout un herméneute du signe? Que faire du rôle d'un lecteur qui, dans le récit policier, devient le double méta-réflexif du sémioticien de la diégèse ? Comment réintroduire cette méta-réflexivité qui, pour le dire avec les mots de Denis Mellier, permet « d'envisag[er] comme un authentique sujet de la fiction le fait de substituer à la question de l'énigme du signifié celle du pouvoir énigmatique du signifiant ${ }^{10}$ ?»

11 Revenons tout d'abord sur cette idée reçue et renouvelée par la critique qui entend faire de l'adaptation l'excuse d'une réintroduction de la hiérarchie texte/image, littérature/ cinéma, culture savante/cultures populaires. Kamilla Elliott rappelle en effet l'importance de l'hybridité texte/image dès les débuts du cinéma, et la centralité de l'illustration dans les magazines périodiques du XIx ${ }^{e}$ siècle. L'exemple de Thackeray pourrait tout aussi bien laisser la place à celui de Doyle, tant les illustrations de Sidney Paget contribuèrent à façonner la représentation collective de Sherlock Holmes. Paget est ainsi à l'origine du couvre-chef du célèbre détective, tout comme William Gillette lui donnera via les planches la pipe recourbée qui deviendra le second signe iconique associé au personnage. Moffat et Gatiss ont en fait recours à l'intermédialité pour réintroduire l'écriture, sous sa forme alphabétique, dans l'image. 
Figure 3 : texte/image $(3,1)$
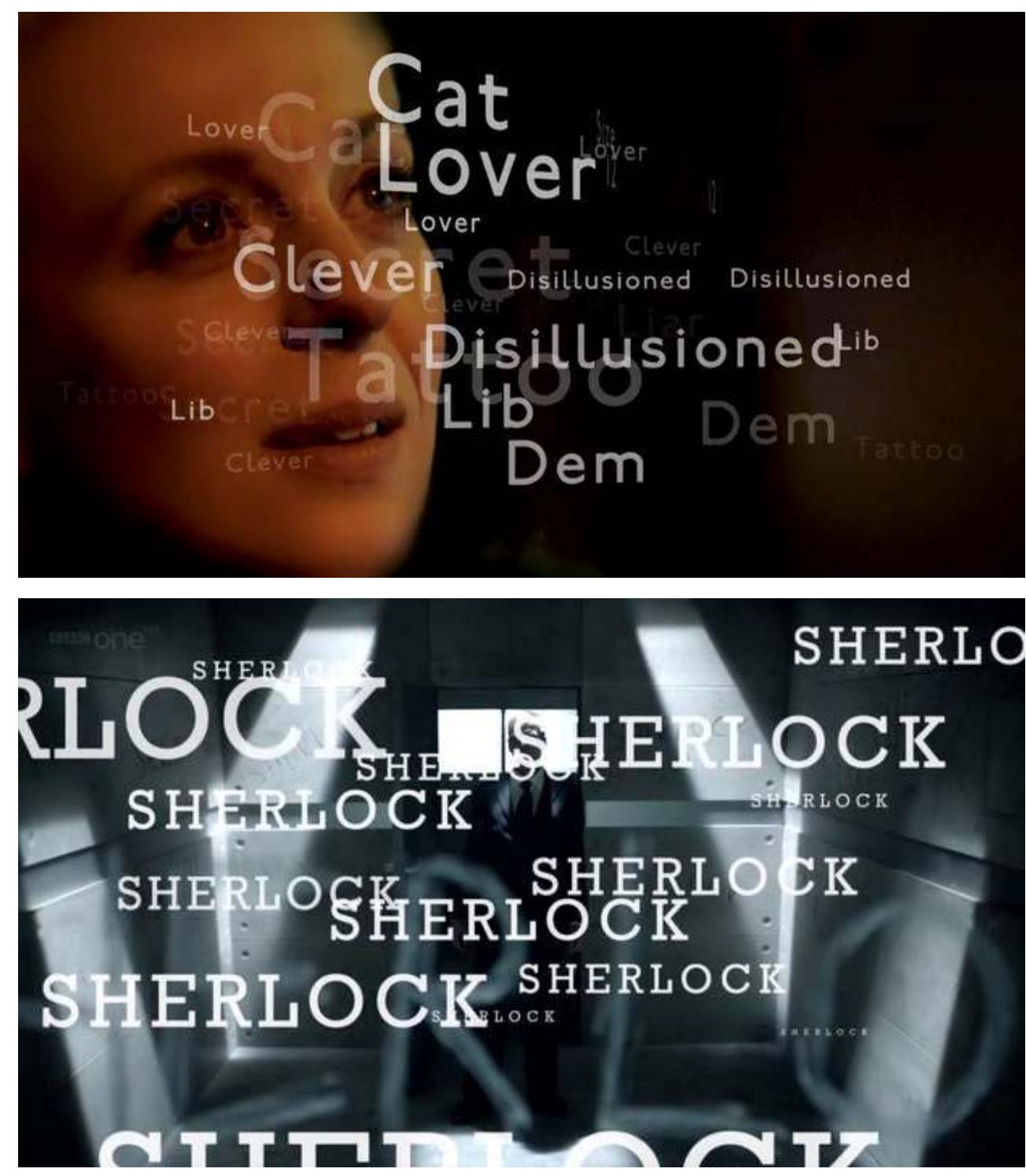

13 Au lieu d'avoir deux temps distincts comme dans les nouvelles qui présentent d'abord la phase d'observation du détective, phase au cours de laquelle le lecteur est dans la même posture d'extériorité que le narrateur, puis la phase où Holmes décrit rétrospectivement les étapes de son raisonnement à un Watson médusé, les réalisateurs de la série BBC proposent une surimposition de mots à l'écran qui permettent au spectateur, et au spectateur uniquement, de suivre «en direct» le fil du raisonnement. Cette technique récurrente apparaît dès la première scène de crime de «A Study in Pink » : 

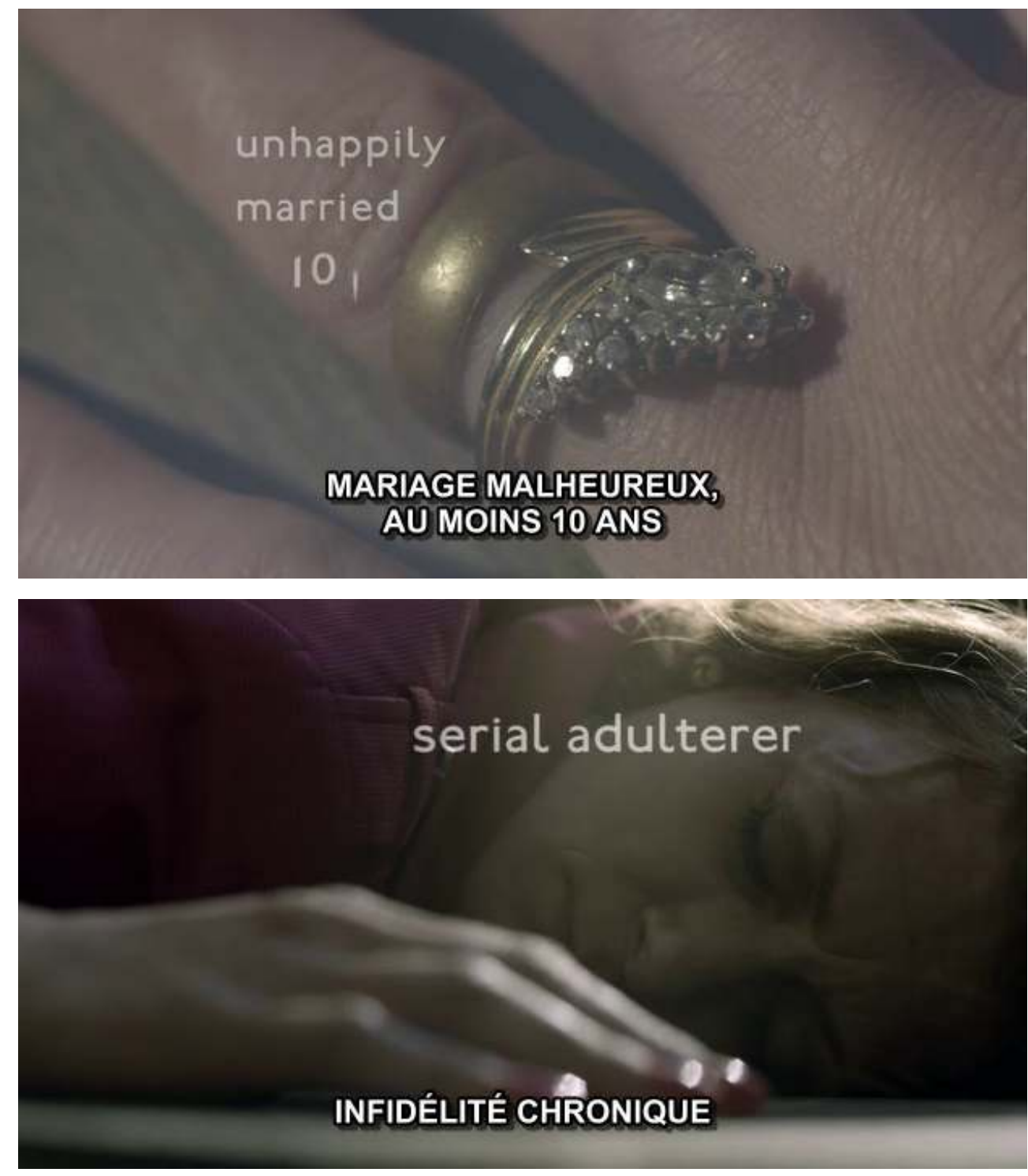

L'intégralité de la séquence montre en outre les ressources sémiotiques propres à l'image. En effet, le rôle de la bande-son, les faux-raccords, les gros plans, travelling avant, panoramiques, accélérés et autres jeux graphiques sur les apparitions/disparitions de mots et de lettres ${ }^{11}$ sont autant de signes donnés au lecteur à décoder pour accéder au raisonnement du détective-herméneute par l'image.

Les mondes diégétiques de Poe et de Doyle étaient des modèles de la suprématie du signe et $\mathrm{du}$ " modèle séculaire de la lecture ${ }^{12}$ » au point de faire du texte la métonymie du monde comme en témoignent la présence de textes cryptés et autres cryptogrammes ${ }^{13}$, vecteurs d'un processus duel d'occultation et de dévoilement, repris dans le second épisode de la saison 1, «The Blind Banker » qui renvoie à « The Dancing Men » ou à « The Musgrave Ritual ${ }^{14} »$.

16 Lettres matérialisées dans l'image par la surerposition, messages codés qui transforment le monde en texte-monde, le modèle de la lecture et l'omniprésence des signes linguistiques et discursifs ne font pas de doute et témoignent d'une intermédialité effective. Mais qu'en est-il du signe symbolique ${ }^{15}$ ? Sherlock est en effet devenu au fil du 
temps une icône à laquelle on associe certains marqueurs symboliques de la figure : la pipe et le chapeau en sont deux exemples. La pipe fait l'objet d'une transposition contextuelle où « a three pipe problem ${ }^{16}$ » devient « a three nicotin patch problem » $(1,1)$, mais le chapeau fait l'objet d'un traitement particulier qui me permet d'introduire la réflexion sur l'image que propose la série.

Le fameux "deerstalker» ne figure pas dans le texte canonique. C'est un accessoire inventé par Sidney Paget. Or, ce n'est pas un hasard si cet attribut unique, et devenu métonymie nécessaire et suffisante du grand détective, apparaît au détour du début de l'épisode 1 de la saison 2 "A Scandal in Belgravia » alors que Holmes et Watson quittent un théâtre et cherchent à se protéger des feux médiatiques. La métaphore théâtrale, introduite dans l'épisode 3 de la saison 1 au sujet de Moriarty ("The curtain rises", $15: 54$ ) devient ainsi le fil rouge d'une saison 2 où théâtralité et mise en scène permettent une réflexion sur l'image et sa circulation dans les médias, les réseaux sociaux et autres espaces numériques. Le chapeau sert donc d'une part la continuité temporelle et historique entre texte canonique et adaptation, mais il permet aussi implicitement de pointer à la fois la part de création à l'œuvre dans le processus et le rôle crucial joué par l'image dans la constitution de la dimension mythique du personnage. Même si notre nouveau Sherlock s'offusque du ridicule de cet attribut en déclarant " This is not my hat ${ }^{17}$ ", c'est bien un méta-discours sur l'image, la représentation et le visuel qui est introduit dans la saison 2.

\section{« Just, don't be dead » $(1: 26: 55,2.3)$}

Figure 5: 2,3

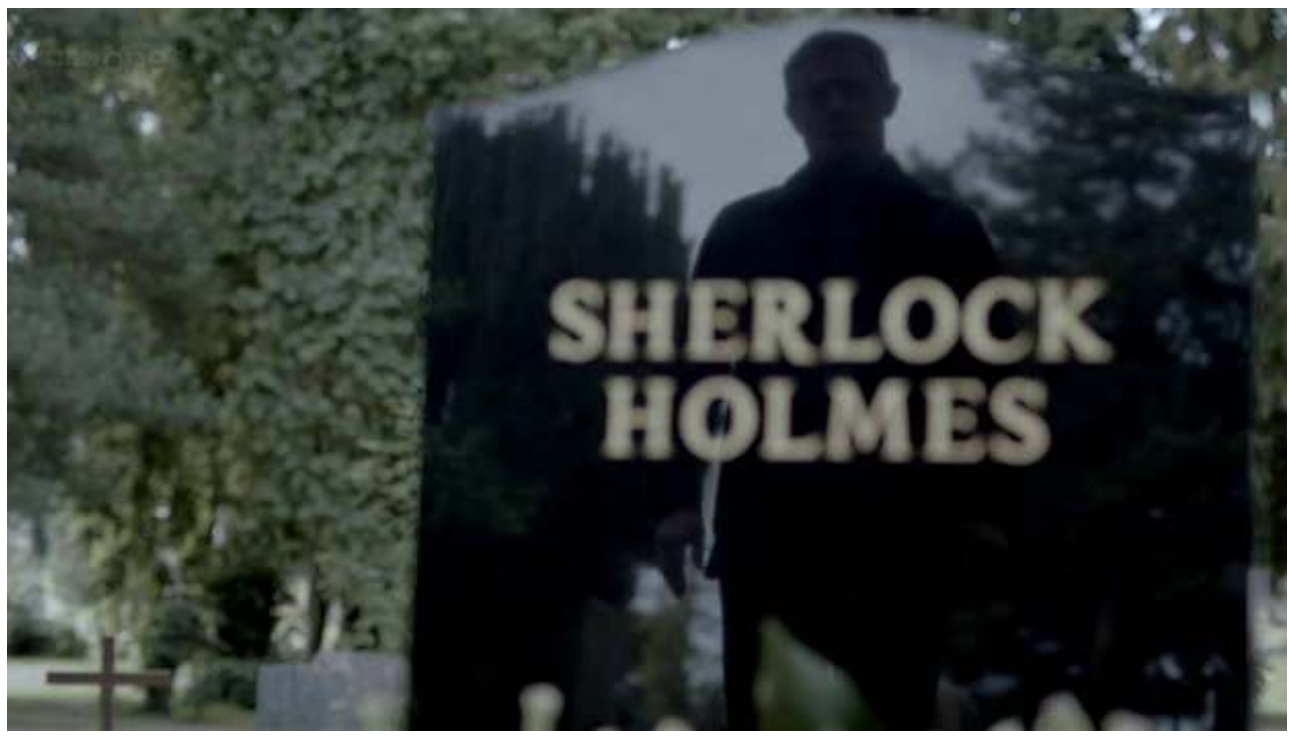

L'adaptation du texte à l'écran peut permettre de révéler certaines « lacunes ", d'exposer une « inquiétude de soi » qui resterait latente dans l'original. Le Sherlock de Doyle était sans conteste un personnage inquiétant car bien qu'acteur principal d'un rétablissement de l'ordre, un nombre certain de facettes du texte original en font une figure de l'altérité 18. Double du criminel, personnage janusien, le détective se constitue également en énigme centrale de l'œuvre de Doyle par l'intermédiaire d'un processus narratif qui ne permet jamais l'accès à ses pensées. 
l'aura inquiétante du personnage et en faire une facette centrale de leur adaptation. Là où la singularité du détective de Doyle était soulignée par le processus narratif, celle de Sherlock est clairement exprimée par des personnages secondaires tels Anderson ou Donovan, et elle redevient donc discursive, par exemple lorsqu'ils le qualifient de psychopathe. Par ailleurs, le comportement décalé de Sherlock par rapport aux normes sociales est souligné à l'envi par Watson, par exemple au début de l'épisode 3 de la saison 2 où Watson décode pour Holmes les signes socio-culturels que le détective s'avère totalement incapable de déceler ${ }^{19}$. On retrouve donc bien la part d'altérité qui était déjà associée au personnage chez Doyle, mais cette dernière est mise à distance par une rhétorique de l'excès comme dans cet exemple :

Figure 6: « Brilliant! Yes! Four serial suicides and now a note. Oh, it's Christmas. » $(16: 27,1,1)$

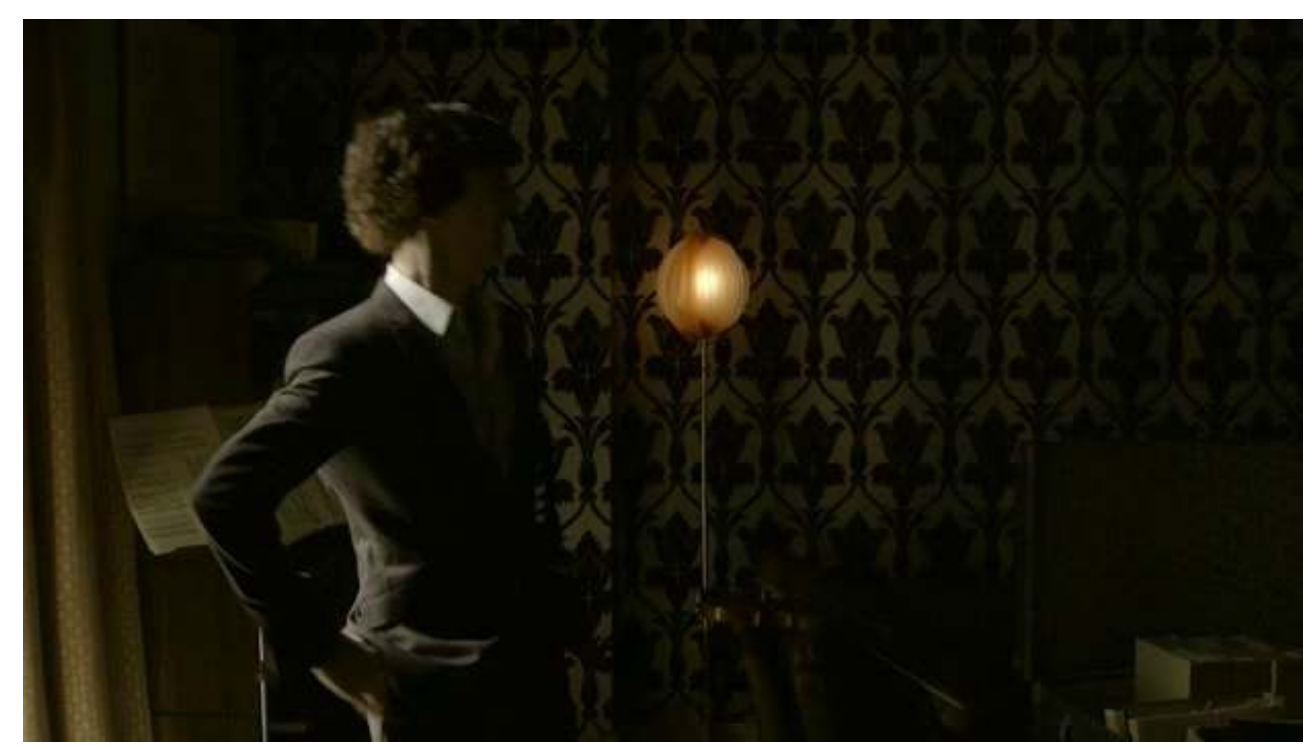

On peut aussi penser au filtre que représente Watson, non plus un prisme narratif comme dans le texte de Doyle, mais un sémioticien des codes et usages sociaux et médiatiques. La mise en abyme du lecteur-sémioticien s'opère donc ainsi toujours par Watson mais par un Watson désormais capable de saisir les signes «linguistique[s], discursif[s] [et] symbolique[s] $]^{20} »$.

Watson dénonce une autre facette de Holmes également liée à l'image de soi, sa propension à se mettre en scène ${ }^{21}:$ «You being all mysterious with your cheekbones, and turning your collar up so you look cool » (« The Hounds of Baskerville», 31 :57). C'est bien le « jeu» du personnage et l'image de soi ainsi produite qui sont implicitement pointés par Watson. Cette fonction du «bloggeur préféré » de Holmes va en s'accentuant dans la saison 2 qui suit l'ascension d'un Sherlock devenu un "internet phenomenon ", dont la notoriété n'a cessé de grandir via les médias: "You're this far from famous ${ }^{22}$ » et qui risque de succomber aux mirages de l'image simulacre. La figure mythique est ainsi mise en danger, dans la fiction, par l'essence même de ce qui la constitue dans le réel, une dimension mythique fondée sur la nécessité de recourir au symbolique et à l'iconique pour exprimer l'impensable d'un hyper-réel devenu simulacre de la réalité.

Le fil rouge de la saison 2 est en effet le soupçon du visuel. Le contrôle de l'information, des images, des réseaux sont les nouveaux outils du pouvoir et les adaptations des deux 
principaux ennemis de Sherlock Holmes chez Doyle, Irène Adler et James Moriarty, en sont la parfaite illustration. Ainsi dans le troisième épisode de la saison 2, "The Reichenbach Fall ", Moriarty devient acteur, metteur en scène et dramaturge de la réalité fictive qu'il construit pour détruire l'image de Holmes. Nous voyons alors poindre une dimension meta-réflexive sans doute plus complexe qu'il n'y paraît. De même, Adler représente, ou incarne littéralement, le leurre du signe dans la performance qu'elle met en scène lors de sa première rencontre avec un Holmes lui même déguisé en prêtre. Adler se présente à lui totalement nue, révélant une totale absence de signe à décoder, et devenant, de fait, une incarnation du sujet désémiotisé, une aporie sémiotique, ce qui permet à Gatiss et Moffat de souligner à nouveau leur jeu méta-réflexif avec une sémiotique de l'image.

Figure 7 : l'aporie sémiotique $(2,1)$

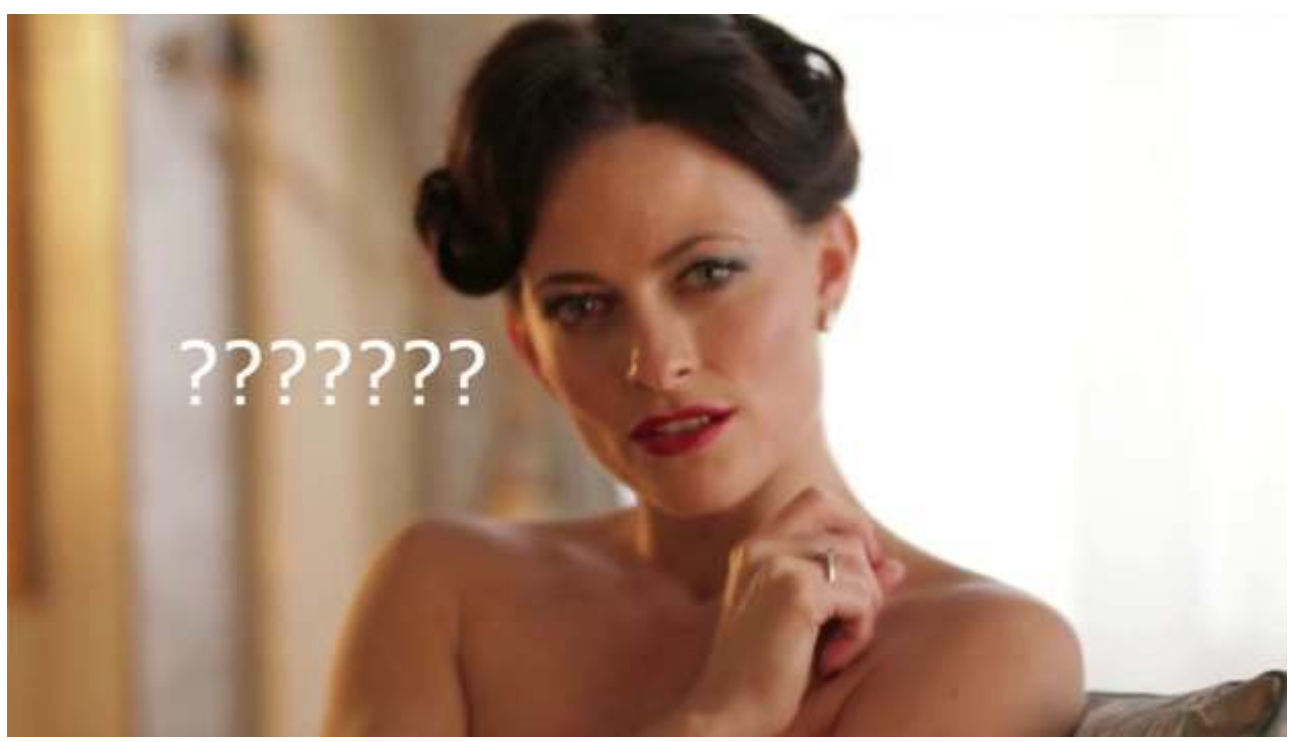

On pourrait dire que les spécificités d'une construction narrative qui repose sur une instance donnant à la fois l'illusion au lecteur de pouvoir battre le détective sur le terrain de l'herméneutique, et bloquant, de fait, la possibilité même d'accéder aux raisonnements méta-abductifs du détective ont été transposées dans une episteme ou le signe et l'image ont remplacé la lettre et la lecture. Moffat et Gatiss revendiquent d'emblée ce que Mellier appelle la «surdétermination » du signe à l'écran ${ }^{23}$ en pratiquant la superposition de mots sur l'image, ou en ayant recours au classique gros plan, comme dans «The Blind Banker » avec le guide de Londres 
Figure 8 : la solution est dans le texte qui apparaît à l'écran $(1,2)$

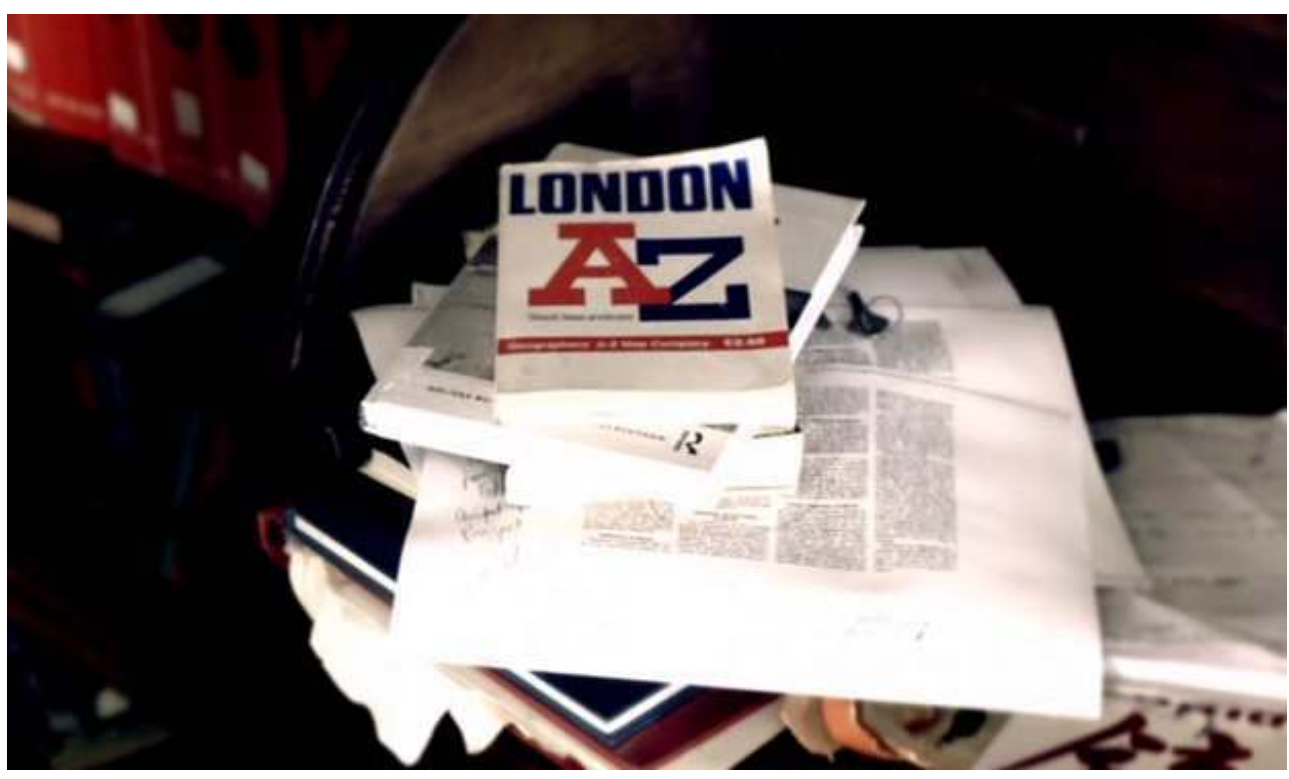

Plus subtilement, la dimension mythique du personnage est également re/déconstruite dans ce transfert d'un médium à l'autre. L'importance de l'image et du cadrage sont soulignés dans les trois saisons qui pratiquent de façon compulsive la mise en abyme de l'encadrement, principalement de Holmes, et, ce, dès le générique d'ailleurs. Filmé de dos devant un cadre (fenêtre et miroir étant les accessoires les plus récurrents), reflet pris dans la lentille d'une loupe, encadrements de face ou de profil, prises de vue qui isolent le sujet dans un cadre lui-même enchâssé dans l'image, les variations sur cette dynamique du cadrage et de l'encadrement qui prend pour sujet de focalisation le détective sont multiples ${ }^{24}$.

Figure 9 : encadrements

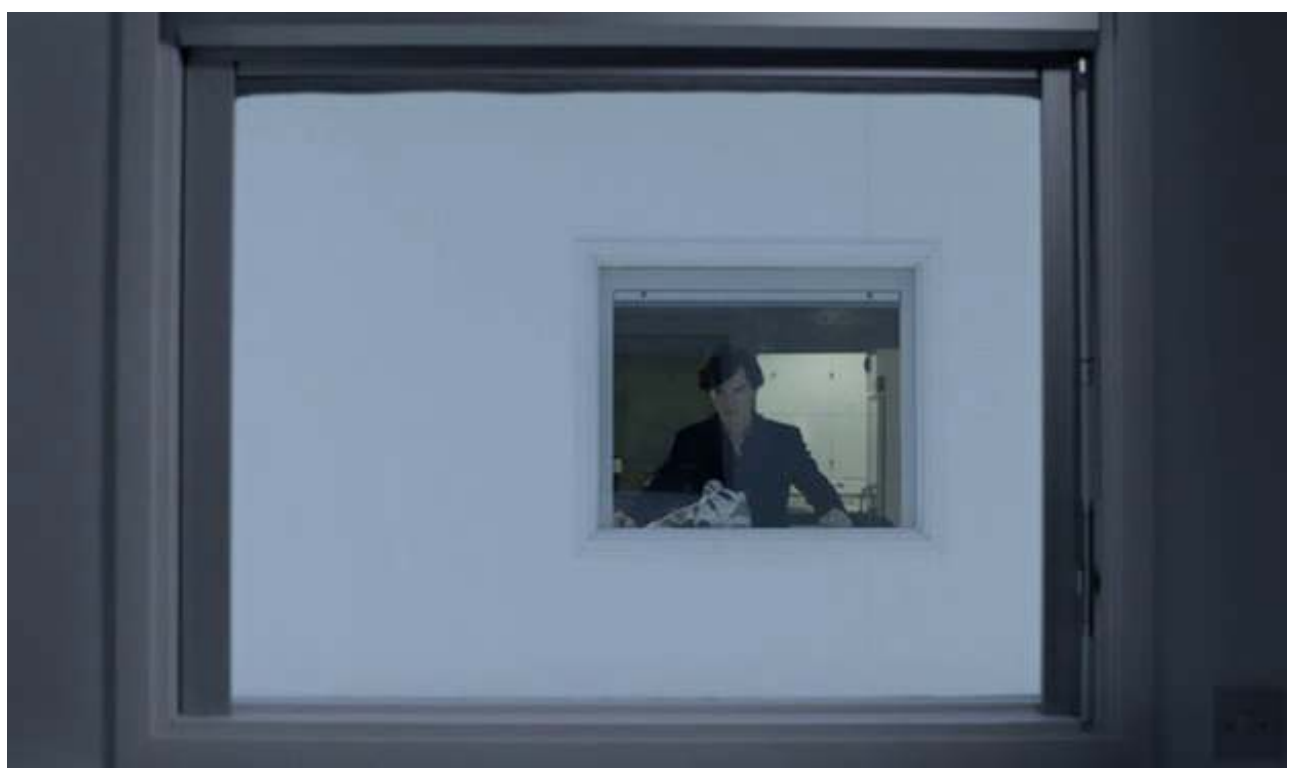



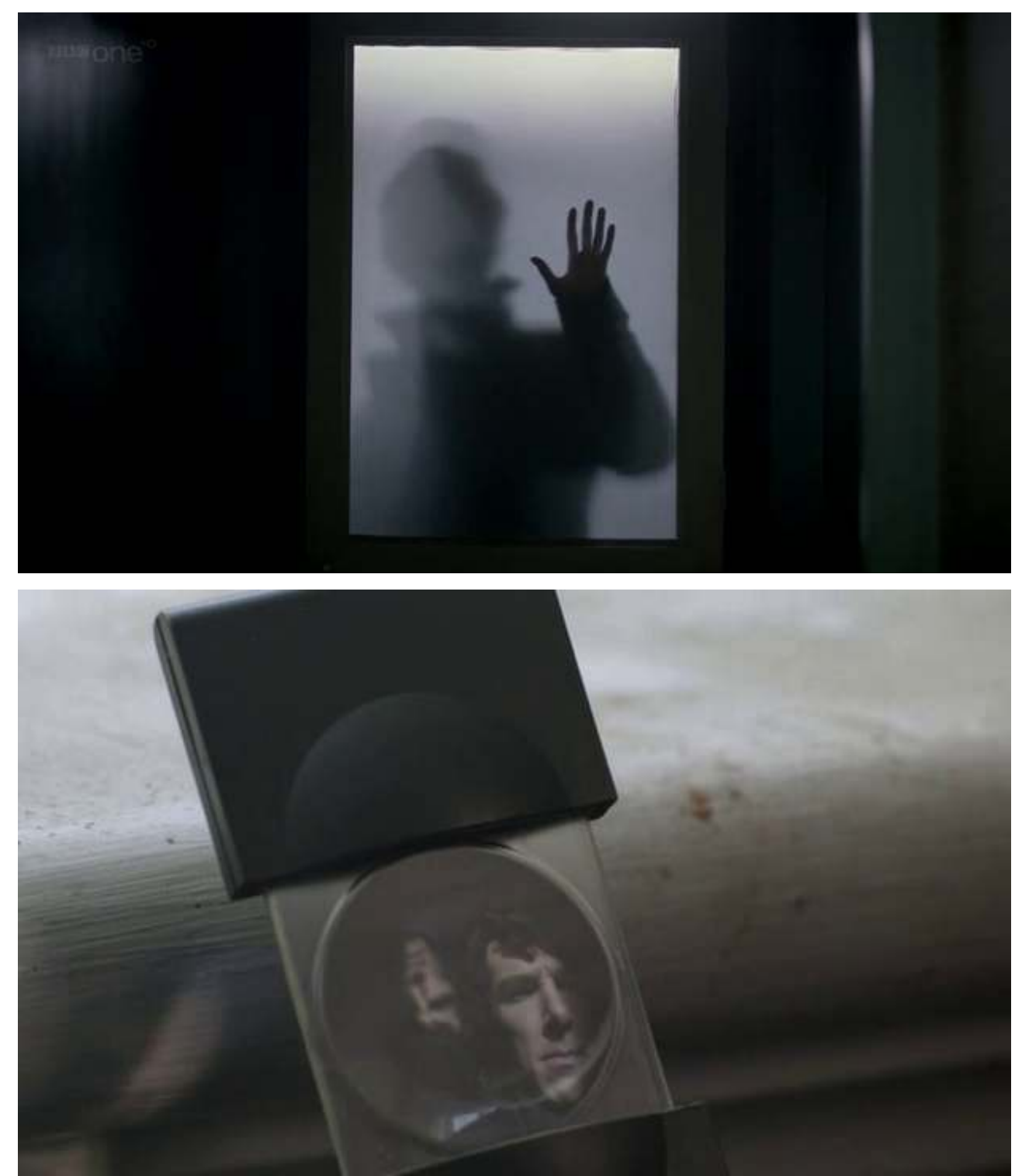

Elles soulignent à la fois les modalités de l'élaboration de la dimension mythique de la figure qui s'est en effet constituée en grande partie d'abord par l'illustration puis par l'adaptation cinématographique, mais elles montrent également que notre episteme est marquée par une culture de l'image qui tend à devenir notre outil exclusif d'appréhension du monde, et là résiderait la nouvelle « inquiétude ».

Il me semble qu'on pourrait aussi y voir la transposition d'une autre facette qui relevait de la construction narrative dans les récits originaux de Doyle. En effet, j’ai déjà montré que le récit traditionnel dans la geste se conclut sur une ekphrasis sémiotique, elle-même souvent mise en exergue par le mini-récit de clôture où Holmes révèle à son public ébahi la solution de l'énigme. Le texte de Doyle avait donc déjà recours à des arrêts sur image, technique proto-cinématographique s'il en est, dans deux situations spécifiques du récit: lors de la révélation finale opérée par le chef d'orchestre Holmes, mais aussi pour mettre en exergue les traits contorsionnés d'un criminel, à l'instar de la description du visage du criminel dans A Study in Scarlet ${ }^{25}$.

Dans les deux cas, l'encadrement était, dans l'original, le symptôme d'une "inquiétude " qui est à l'origine de l'accession de la figure au statut de figure mythique. Notons cet 
encadrement terrifiant du visage figé d'un criminel dans un contexte de crise épistémologique post-théorie de l'évolution où l'on s'interroge sur un atavisme en matière de criminalité, où Lombroso compare crânes de criminels et crânes de singe, où Bertillon met au point l'anthropométrie, où le besoin de se représenter l'homme originel n'a d'égal que la répulsion qu'il déclenche comme le donne à montrer les multiples caricatures de l'époque ${ }^{26}$ :

Figure 10

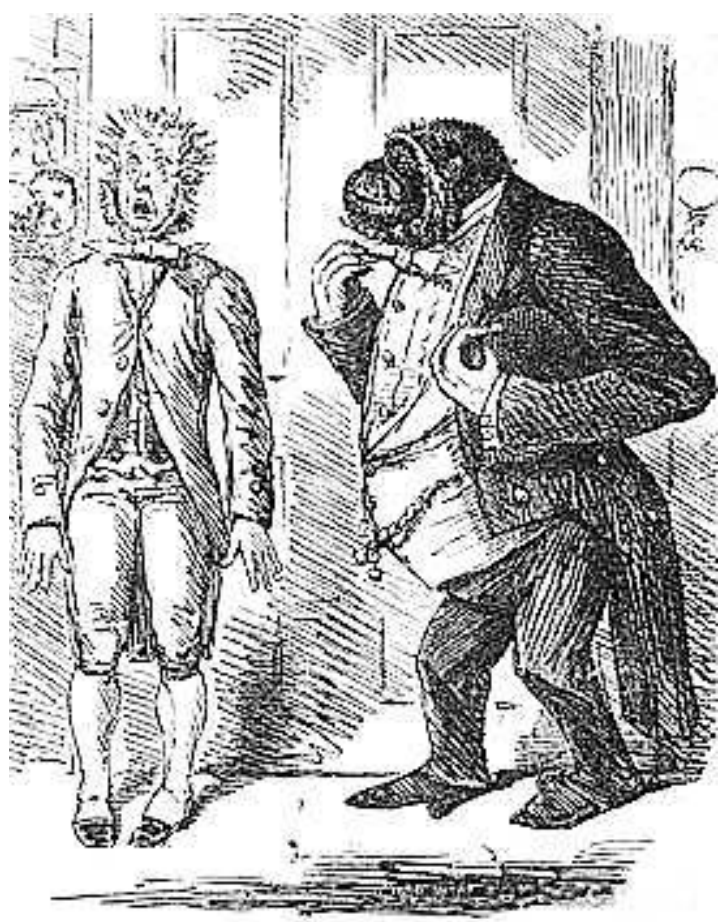

Anonym. Mai 1861

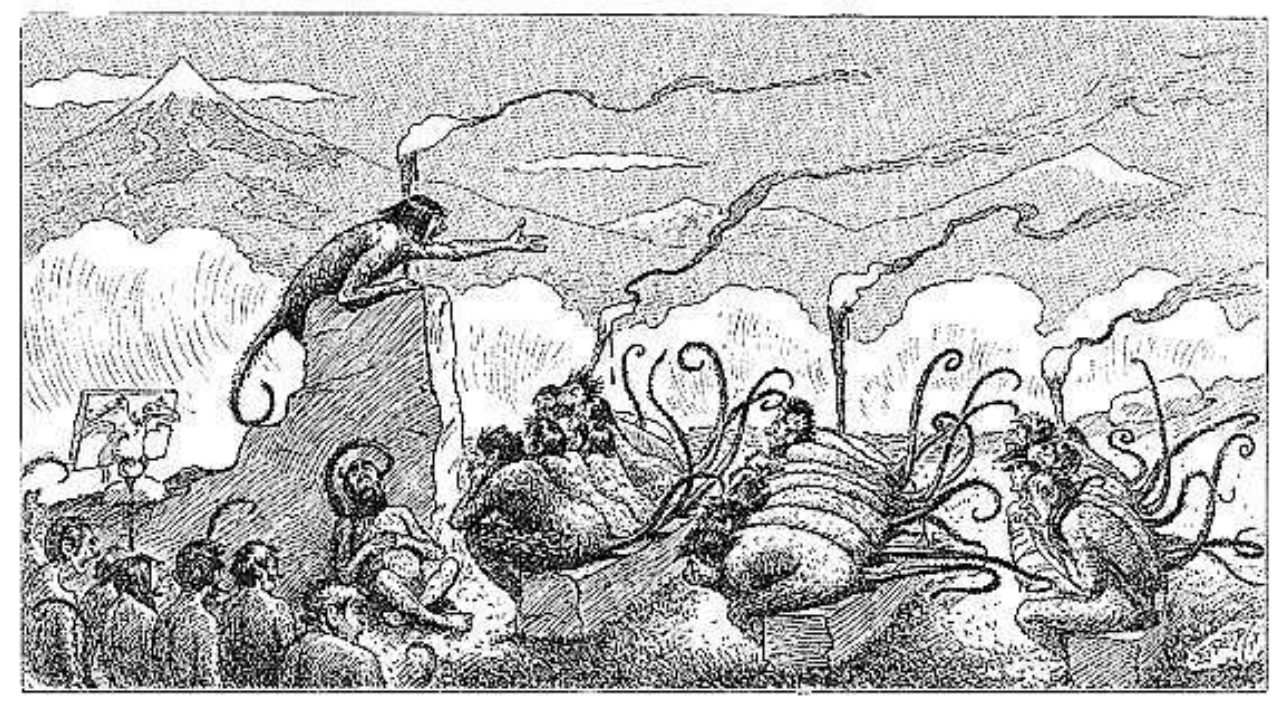

PREHISTORIC PEEPS.

A NIGIIT LECTLBE OX EROLUTIOX.

E.T Reed, 1860 
alors possible d'avancer qu'à cet encadrement du visage des criminels correspond plutôt en clôture de récit, le mini-récit conclusif qui apporte solution et résolution au mystère exposé en début de texte, et rassemble les fragments épars en une image cohérente de la réalité. Dans les deux cas, les techniques de l'encadrement et de l'enchâssement narratifs exposent et tentent en même temps de pallier l'inquiétude contextuelle relative à la représentation iconique de l'origine de l'être humain.

Avec Sherlock, cette récurrence du motif de l'encadrement ne change pas mais elle sert une autre visée. Elle contribue, selon moi, à la fois à rappeler que le modèle visuel prévaut dans la nouvelle culture de l'écran ${ }^{27}$, que la place de l'humain dans ce nouveau cadre n'est pas encore stabilisée, et que cette nouvelle sémiologie est effectivement davantage liée à l'espace qu'au temps ${ }^{28}$.

\section{Figure 11 : omniprésence de l'écran}
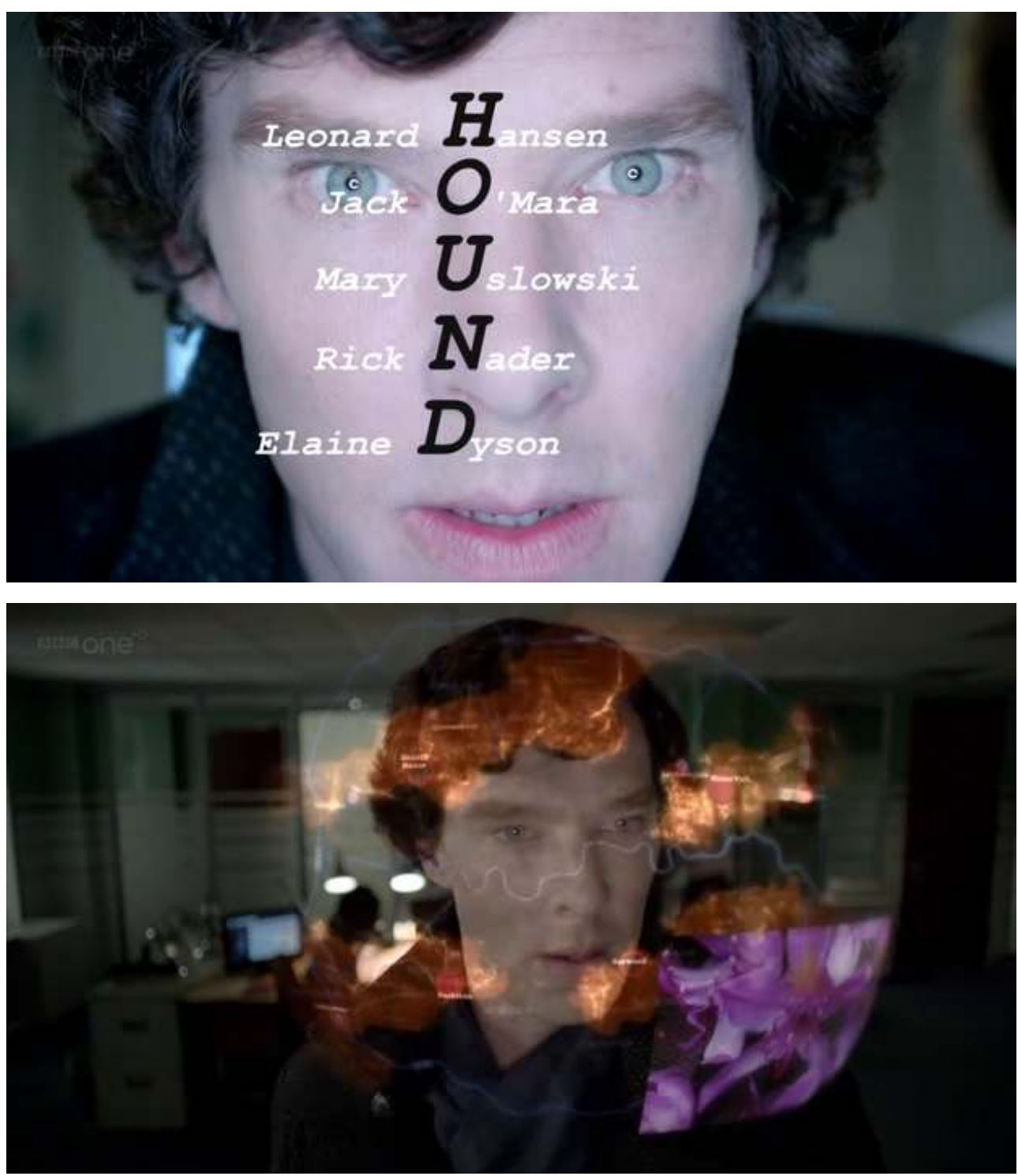

Les interrogations sur les origines de l'espèce ne sont en effet plus guère d'actualité de nos jours. En revanche, les devenirs de l'humain dans une société bio-technologique et écranique sont au cœur de la réflexion contemporaine sur le posthumain et les 
subjectivités numériques. Aussi, la série propose-t-elle également une réflexion sur le pouvoir des images et des médias, sur l'image et les simulacres de réalité qu'elle peut construire, et mène ainsi le spectateur vers des questionnements relatifs à la porosité entre deux espaces, l'espace de la réalité et l'espace de la fiction ${ }^{29}$.

Le nouveau Sherlock est sans surprise un détective connecté qui a sans arrêt recours à la toile et autres réseaux numériques, sociaux ou non. La mise en abyme des écrans se poursuit donc et ils deviennent le symbole d'une nouvelle culture de l'écran qui est mis en image. Là encore, toute la spécificité du médium est convoquée par Moffat et Gatiss et ils vont bien plus loin qu'une simple mise en abyme. On peut en effet s'arrêter sur deux exemples, celui de la course-poursuite du taxi dans «A Study in Pink» et celui du « mind palace ».

"A Study in Pink» reprend en effet la course-poursuite dans Londres qui, dans le canon se déroule dans «The Sign of Four $»^{30}$. Dans cet exemple ${ }^{31}$, c'est l'esprit de Sherlock qui devient une sorte de GPS avec écran permettant de suivre le parcours (pour Sherlock et en abyme pour le spectateur). L'intérêt de cet extrait est de montrer que ce sont les images mentales du détective qui sont transposées en images numériques. Nous entrons alors de plain-pied dans ce que Bertrand Gervais appelle une «image opératoire, une image qui permet d'intervenir sur le monde [...] le monde réel, tangible », ce qui modifie le statut de l'image :

Son rapport au monde et aux objets [...] n'est plus iconique ni indiciel, il est devenu performatif. L'image ne témoigne plus d'une présence [...], mais elle agit sur cette présence et entreprend de la modifier. En culture de l'écran, l'image permet une intervention sur le monde ${ }^{32}$.

33 Ce que Holmes appelle son «mind palace $»^{33}$ est un second exemple de la recontextualisation et du transfert opéré d'une episteme post-darwinienne à notre culture de l'écran. L'une des caractéristiques de cette culture de l'écran que pointe Gervais est la hantise du « morcellement du sensible » à laquelle répondrait une « logique des flux » qui s'inscrit dans la matérialisation d'une fluidité, d'une interactivité de l'interface écranique qui vise à faire disparaître cette dernière en tant que dispositif afin de lui conférer le statut de " processus de connaissance » :

[Cette image] devenue processus de connaissance [...] on la regarde, on la manipule, on s'en sert pour connaître et se reconnaître, et son régime sémiotique singulier impose sa logique associative et relationnelle ${ }^{34}$.

34 À la manière d'Anderton dans Minority Report ${ }^{35}$ lorsqu'il devient un chef d'orchestre de l'image sur fond de $8^{\mathrm{e}}$ symphonie de Shubert, Holmes manipule des images, non plus produites par des « precogs » mais stockées dans son palais mental. 
Figure 12: «Le palais mental »

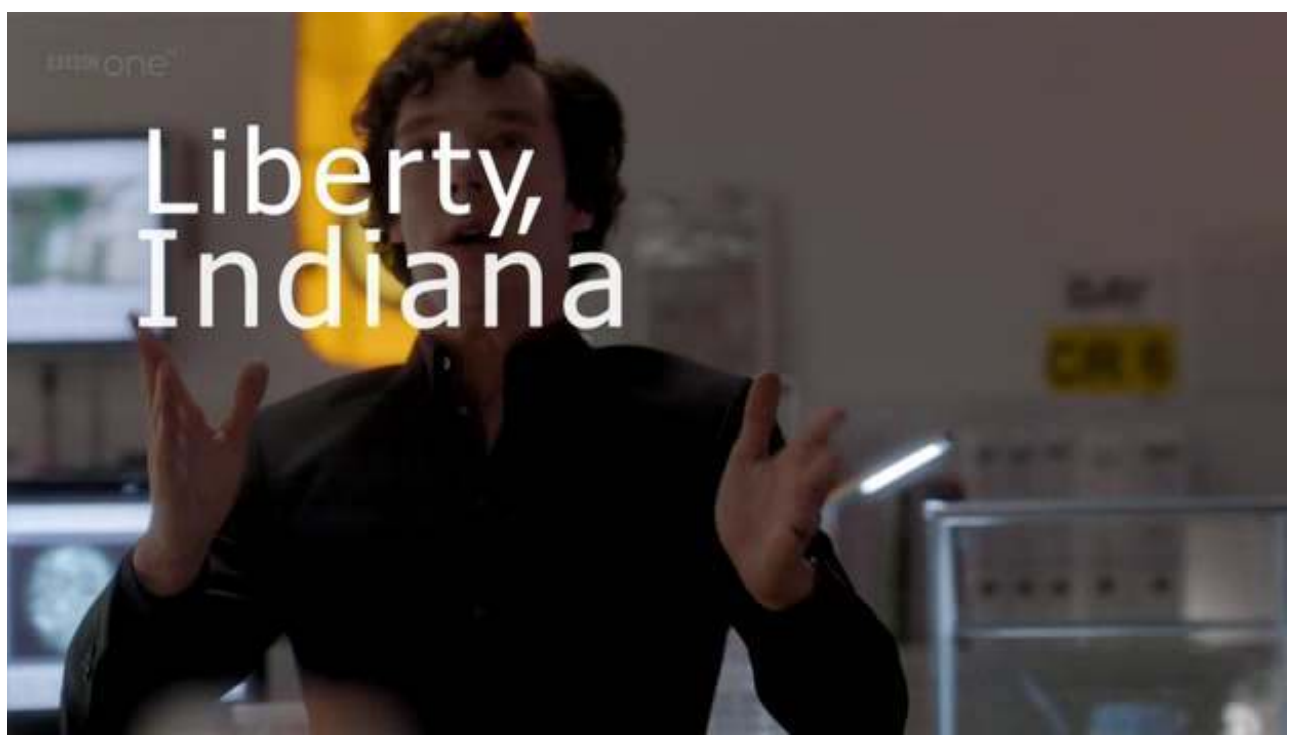

La logique est bien associative et relationnelle, et l'image manipulée s'inscrit dans une visée performative d'intervention sur le monde. L'interface écranique en tant que dispositif disparaît également puisque l'écran intérieur de ce palais mental se fond avec le dispositif de l'écran qui sépare spectateurs et univers fictif de la diégèse. Holmes regarde l'écran de son palais mental dans une position symétrique et en miroir de celle du spectateur (sauf qu'il s'agit là encore d'une illusion car cela impliquerait que les mots apparaissent à l'écran de gauche à droite). Le « mind palace » correspondrait donc à une transposition à l'écran de l'ekphrasis sémiotique qui apportait clôture au processus narratif mis en place par Doyle. La subtilité de la transposition réside dans l'adaptation à la fois au médium spécifique qu'est l'image et au contexte de la culture de l'écran. Images et mots deviennent des opérateurs analogues qui participent à une intervention sur le monde fictif renvoyant à son tour, par disparition de l'interface (ce que voit Holmes est ce que nous voyons), à une correspondance entre les deux côtés de l'écran et à une porosité entre fiction et réalité. Chef d'orchestre, maître du jeu, Holmes devient donc aussi manipulateur d'images, «I prefer to do my own editing », déclare-t-il d'ailleurs à Kitty, la journaliste manipulée par Moriarty dans «The Reichenbach Fall». A la correspondance entre détective-sémiologue et lecteur-sémiologue de la lettre qui avait été établie par Poe et Doyle, Moffat et Gatiss substituent un détective et des spectateurs qui reprennent toutes les déclinaisons d'une sémiotique du texte transposées en sémiotique de l'image. Ainsi, à la fin de la saison 2, Holmes orchestre sa propre mort ${ }^{36}$ et le «montage » de ce que Watson, synecdoque du spectateur, va voir et croire. Le jeu est dès lors lancé et il peut désormais s'étendre en dehors de la fiction.

Sherlock Holmes est devenu une figure mythique car, pour reprendre la définition de Jean-Jacques Lecercle ${ }^{37}$, il représentait un compromis permettant de résoudre temporairement une tension résultant d'une crise ontologique déclenchée par l'effet iconoclaste qu'eut la théorie de l'évolution sur les représentations iconiques de l'humain. L'obsession de la trace et du signe étaient alors directement liée à une perte de sens et de direction du texte-monde. Moffat et Gatiss ont su transposer ce contexte à celui de l'ère post-cybernétique, celle de l'homme augmenté par les bio-technologies et les 
technologies du numérique, celle de la théorie de l'information où le texte-monde a muté en un monde de l'image.

Le jeu avec l'image se déploie dès lors dans plusieurs directions. Jeu avec le texte d'origine, bien sûr, mais aussi jeu avec des effets de fiction spécifiques à l'original qui ont contribué à l'élaboration de la figure mythique en lui conférant une autonomie dans la fiction. Cela dit, le jeu devient plus subtil lorsqu'il relève d'une pratique de la distanciation par la référence. Ainsi, Holmes, esprit encyclopédique, herméneute du texte-monde capable de rétablir du sens, devient Sherlock, dont l'esprit est un disque dur ${ }^{38}$, qui manipule les images tel un nouveau démiurge, une subjectivité numérique qui fait presque disparaître l'interface écranique et transforme les images en processus de connaissance inscrit dans une fluidité maximale, typique de la culture de l'écran ${ }^{39}$. Figure d'une subjectivité numérique en grande partie déshumanisée, le détective démontre sa capacité d'adaptation à un nouveau contexte de crise épistémologique et sa propension à cristalliser les tensions ontologiques propres à une episteme où la rhétorique de l'image et les simulacres du visuel sont les nouveaux vecteurs d'une « inquiétude de soi ».

Tous ces aspects semblent attester d'une grande maîtrise de Gatiss et Moffat dans l'art de l'adaptation et dans celui de la transposition. Plus intéressant encore : le renouvellement du phénomène de contamination de la réalité par la fiction. Comme lors de ce que l'on nomme le "grand hiatus ", c'est-à-dire les 10 années au cours desquelles Holmes est « mort » avant de faire son retour en 1903 dans « The Empty House », l'année qui a séparé la diffusion de «The Reichenbach Fall » de celle de «The Empty Hearse » a été l'occasion d'une créativité étonnante dans les communautés de fans sur les réseaux internet. «We believe in Sherlock », " Tumbler » et consorts se sont littéralement emparés de la matière fictive et les scénarios sur la mise en scène du suicide de Sherlock ont proliféré sur la toile. A l'ère de l'interactivité numérique, dans une société écranique où les interfaces tendent à disparaître, les créateurs de Sherlock ont immédiatement adapté ce phénomène dans "The Empty Hearse », transformant ainsi la fiction en écho du réel et donnant un tour de vis supplémentaire à la porosité entre fiction et réel, et à l'aura mystérieuse de ce nouveau manipulateur d'images puisqu'aucun des scénarios présentés dans la fiction n'est validé... par la fiction.

\section{BIBLIOGRAPHIE}

CRIGNON Hélène, «L'énigme Sherlock Holmes », La Licorne, n 64, 2006, http://licorne.edel.univpoitiers.fr/sommaire.php?id=2900. (25/02/15)

ESQUENAZI Jean-Pierre, Les séries télévisées, l'avenir du cinéma ?, Paris, A. Colin, 2014

GELLY Christophe; "The Hound of the Baskervilles Revisited: Adaptation in Context", Arthur Conan Doyle, OScholars special issue, edited by Sarah E. MAIER, 2015, http://oscholars-oscholars.com/ special-issues/doyle/, consulté le 20/09/2017.

GERVAIS Bertrand, « Sommes-nous maintenant/It is now ? », Conférence donnée au colloque Figura d'avril 2014, http://oic.uqam.ca/fr/print/56656. 
HUTCHEON Linda, A Theory of Adaptation, NY \& London, Routledge, 2006.

JEANNELLE Jean-Louis, « Rouvrir le débat sur l'adaptation : Kamilla ELLIOTT et les rapports entre le roman et le cinéma », 2010, http://www.fabula.org/lodel/acta/document.php?id=5632, consulté le $20 / 09 / 2017$.

LECERCLE J-J, « Dracula : une crise de sorcellerie », Dracula, Stoker-Coppola, eds. Gilles MENEGALDo et Dominique SIPIERE, Paris : Ellipses, 2005, p. 9-29.

Machinal Hélène, Conan Doyle, Rennes, Presses Universitaires de Rennes, 2004.

MACHINAL Hélène, «Le singe et l'ange : le corps de l'origine dans la littérature de la fin du XIX siècle ", Savoir médical et représentation du corps humain XVII-XIXe siècles, Talairach, L. \& Mandressi, R. (eds.), Revue en ligne Epistemo-critique, 2012, www.epistemocritique.org. NAUGRETTE J-P, « Sherlock (BBC 2010) : Un nouveau limier pour le $\mathrm{XX}^{\mathrm{e}}$ siècle ? », Études Anglaises 64.4, 2011, p. 402-414.

MELLIER Denis, «L'impossibilité filmique de l'énigme policière », in MÉNÉGALDO, G. \& SIPIÈRE, D. (eds.), Les récits policiers au cinéma, La Licorne, Hors série - Colloque VIII, Poitiers, 1999, p. 9-24. ROPARS-WUILLEUMIER Marie Claire, Ecraniques ; le film du texte, Presses Universitaires de Lille, 1990. STEIN L. E. et K. BUSSE (eds.), Sherlock and Transmedia Fandom: Essays on the BBC Series, Jefferson, North Carolina, McFarland, 2012.

Sherlock, Moffat \& Gatiss, BBC, 2010- (3 saisons)

Minority Report, Spielberg, 2002.

\section{NOTES}

1. Phénomène marquant de ces dernières décennies, l'essor fulgurant de cette nouvelle facette de la culture populaire remonte cependant à l'introduction de la télévision dans les foyers. Voir Jean-Pierre Esquenazi, Les séries télévisées, l'avenir du cinéma?, Paris, A. Colin, 2014, chapitre 1.

2. Sur l'incipit, on pourra aussi consulter Naugrette, «Sherlock (BBC 2010) : Un nouveau limier pour le xx siècle? », Études Anglaises 64.4, 2011, p. 402-414.

3. De même, le souci du détail qui va caractériser cette adaptation apparaît immédiatement. Stamford et Watson ne déjeunent plus ensemble au Criterion de Londres, mais ils boivent un café dans un gobelet en carton de vente à emporter du Criterion.

4. Marie Claire Ropars-Wuilleumier, Ecraniques. le film du texte, Lille, Presses Universitaires de Lille, 1990, p. 163.

5. Christophe Gelly, "The Hound of the Baskervilles Revisited: Adaptation in Context ", Arthur Conan Doyle, OScholars special issue, edited by Sarah E. Maier, http://oscholars-oscholars.com/ special-issues/doyle/

6. Voir le début de saison 3, épisode 2.

7. «The Adventure of the Abbey Grange ». On notera également la tendance à l'auto-dérision qui caractérise cette nouvelle adaptation lorsque dans l'épisode 2 de la saison 3, Holmes et Watson enterrent la vie de garçon de ce dernier et se retrouvent à enquêter alors qu'ils sont pour le moins saouls. On voit alors Holmes péniblement essayer de se souvenir de cette phrase emblématique.

8. "You really are an automaton, a calculating machine," I cried. The Sign of Four

9. L'analyse des signes, traces et détails est en effet le fondement de la méthode du détectiveherméneute depuis Dupin et le leurre narratif du récit policier consiste à donner à la voix 
narrative une double fonction contradictoire. Watson est notre accès narratif aux pensées du détective mais ce n'est là qu'illusion car l'esprit de Holmes doit rester inaccessible sans quoi il n'y a plus ni plaisir ni moteur au texte.

10. Denis Mellier, «L'impossibilité filmique de l'énigme policière », in G. Ménégaldo \& D. Sipière (eds.), Les récits policiers au cinéma, La Licorne, Hors série - Colloque VIII, Poitiers, 1999, p. 15 [p. 9-24].

11. Voir par exemple l'analyse des vêtements de l'envoyé de Buckingham Palace dans «A Scandal in Belgravia ».

12. L'expression vient de Denis Mellier, «L'impossibilité filmique de l'énigme policière », art. cit., p. 13.

13. Chez Poe avec «The Gold Bug ", comme chez Doyle avec "The Dancing Men » et "The Five Orange Pips ».

14. Voir H . Machinal, Conan Doyle, Rennes, Presses Universitaires de Rennes, 2004, p. 43-46.

15. On peut rappeler ici « [...] le modèle hérité de Lessing servit à établir que le roman est par essence conceptuel, linguistique, discursif, symbolique, autrement dit lié au temps, alors que le film est perceptif, visuel, représentationnel, littéral, bref, lié à l'espace » Jean-Louis Jeannelle, "Rouvrir le débat sur l'adaptation: Kamilla Elliott et les rapports entre le roman et le cinéma », http://www.fabula.org/lodel/acta/document.php?id=5632 (consulté le 15/01/15)

16. Arthur Conan Doyle «The Red-Headed League ».

17. Voir aussi le début de l'épisode 3 de la saison 2 ( $3: 23-3: 55)$ et l'échange sur le chapeau qui se termine par : «I mean, this isn't a deerstalker now. It's a Sherlock Holmes hat. »

18. Voir Machinal, op. cit., p. 129-155 et H. Crignon, «L'énigme Sherlock Holmes », La Licorne, n ○ 64, http://licorne.edel.univ-poitiers.fr/sommaire.php?id=2900.

19. Cet aspect était totalement absent du texte de Doyle où Holmes est même présenté comme capable de faire preuve de beaucoup de psychologie et d'attention à l'autre lors de ses échanges relatifs à l'enquête avec un client, un suspect ou un témoin.

20. Jeannelle, op. cit.

21. « He does love to be dramatic », déclarait déjà Mycroft dans « A Study in Pink » (37:02)

22. Voir Saison 2, épisode 3, tout le début de l'épisode. http://www.planetclaire.org/quotes/ sherlock/series-two/the-reichenbach-fall/ (23/02/15)

23. Mellier, op. cit.

24. On peut évoquer ici le rapprochement que propose Esquenazi entre séries et variations. Nous aurions ainsi au fil des épisodes et des saisons des variations, ou une série de portraits encadrés du détective. Esquenazi, op. cit., p. 27.

25. «On his rigid face there stood an expression of horror, and as it seemed to me, of hatred, such as I have never seen upon human features. This malignant and terrible contortion, combined with the low forehead, blunt nose, and prognathous jaw gave the dead man a singularly simious and ape-like appearance, which was increased by his writhing, unnatural posture. I have seen death in many forms, but never has it appeared to me in a more fearsome aspect than in that dark grimy apartment, which looked out upon one of the main arteries of suburban London. " Doyle, A Study in Scarlet, ch. 3, https://sherlock-holm.es/stories/pdf/a4/1sided/stud.pdf (consulté le 15/03/15)

26. H. Machinal, «Le singe et l'ange : le corps de l'origine dans la littérature de la fin du XIX siècle ", Savoir médical et représentation du corps humain XVII-XIX ${ }^{e}$ siècles, L. Talairach \& R. Mandressi (eds.), Revue en ligne Epistemo-critique www.epistemocritique.org.

27. Bertrand Gervais, "Sommes-nous maintenant/It is now ? ", Conférence donnée au colloque Figura d'avril 2014, http://oic.uqam.ca/fr/print/56656. (consulté le 07/03/15)

28. Voir Jeannelle, op. cit. On pourrait en effet montrer plus en détail que le texte de Doyle correspond bien à une organisation narrative qui se déploie d'abord dans le temps tandis que les adaptations à l'écran (grand ou petit) ont davantage recours à l'espace. Cela dit, ce point 
mériterait plus ample développement si l'on évoque l'épisode 2 de la saison 3, qui joue sur les flashbacks pour construire à la fois le récit du crime et celui de l'enquête.

29. Ainsi, dans la figure 11, capture de gauche, le regard porté sur Sherlock est situé à «l'intérieur » de l'ordinateur (et donc de l'écran). Est-ce alors le spectateur qui devient l'œil... de la caméra?

30. On trouve une seconde course-poursuite fonctionnant sur la même assimilation de l'esprit du détective à un navigateur GPS dans « The Empty Hearse ».

31. Voir « A Study in Pink », 52:00-54:03.

32. Gervais, op. cit., p. 12. http://oic.uqam.ca/fr/print/56656 (19/03/15)

33. Voir en particulier « The Hounds of Baskerville », 1:11:24-1:11:26.

34. Gervais, op. cit., p. 10.

35. Minority Report, Spielberg, 2002.

36. Il joue encore une fois sur le sens de la lettre en assimilant son appel téléphonique à la note habituellement laissée par un suicidé alors que sa véritable manipulation concerne littéralement la mise en scène de ce faux suicide.

37. Lecercle part de Lévi-Strauss et pose qu'une figure mythique constituerait « une solution imaginaire à une contradiction réelle, un opérateur logique pour concilier deux propositions qui se contredisent et forment une "double contrainte" collective », J-J Lecercle, " Dracula : une crise de sorcellerie", Dracula, Stoker-Coppola, eds. Gilles Menegaldo et Dominique Sipiere, Paris: Ellipses, 2005, p. 10 [9-29].

38. Voir par exemple: « The Sign of Three », 1:13:07.

39. Voir aussi Saison 3, épisode 1, 45 :55-46:34 pour des remarques similaires sur la dichotomie entre morcellement du sensible et logique des flux.

\section{RÉSUMÉS}

Depuis la publication des nouvelles dans le Strand en 1887, les textes de Doyle ont donné lieu à de très nombreuses adaptations d'emblée marquées par leur caractère transmédiatique ; on pense à l'adaptation de Gillette au théâtre ou aux premiers films muets. Il est étonnant de noter que cette adaptabilité du texte littéraire ne s'est pas démentie depuis, les dernières en date parmi les plus populaires étant deux séries TV, Elementary et Sherlock.

Il y a donc lieu d'analyser les attributs qui permettent cette porosité entre les siècles, les contextes, mais aussi des médias comportant des traits distinctifs, en particulier du fait d'un passage du texte à l'image qui, dans le cadre des analyses narratologiques du récit policier, n'est pas sans poser question. Nous repartons donc de la fonction attributive des objets et de leur rôle dans l'élaboration de la figure mythique pour nous attacher ensuite à la dynamique de l'adaptation dans la série de Moffat et Gatiss et plus particulièrement à la distanciation par la dynamique de la dérision et de l'ironie. Que nous révèle cette dernière du regard que porte cette nouvelle adaptation sur la figure mythique? Enfin, nous nous attarderons sur les raisons d'une dichotomie entre continuité et rupture à l'œuvre dans la série, une tension qui trouve son origine dans le canon. La porosité entre fiction et réalité est en effet l'une des caractéristiques associée à Sherlock Holmes depuis le XIX et en dépit des variations repérables, cette facette persiste au XXI e avec le « new sleuth » que nous livre la BBC. 
Since 1887 and the publication of the Sherlock Holmes stories in the Strand, Doyle's texts have been adapted, and those adaptations have been transmediatic, as exemplified in the early days of the process by Gillette's play. The adaptability of the literary text has been continuous ever since. The last shows worth noting in that sense are TV series: Elementary and Sherlock.

The point of this article will be to analyse the attibutes which allow such porosity between centuries, contexts, but also dictinct media, especially as adaptation from text to film raises questions within the narrative context of detective novels. Thus we will first focus on objects and attributes of the mythical figure of the detective to analyse the role they play in the making of that mythical dimension, before tackling adaptation as such in Moffat and Gatiss' production, a fiction marked by irony and derision. What effect does such an ironic prism create on adaptation? Finally, we will ponder the dichotomy between continuity and rupture and the interaction between fiction and reality, a persisting facet of the canon since the $19^{\text {th }}$ century which perdures with the new sleuth of the BBC Series.

\section{INDEX}

Keywords : Holmes Sherlock, adaptation, irony, fiction, reality, Sherlock (BBC)

Mots-clés : Holmes Sherlock, adaptation, ironie, Sherlock (BBC), fiction, réalité

\section{AUTEUR}

\section{HÉLÈNE MACHINAL}

Hélène Machinal est professeur à l'Université de Bretagne Occidentale et membre de HCTI (EA 4249). Elle est spécialiste de littérature fantastique, du roman policier et de la fiction spéculative au XIX et au XX ${ }^{\mathrm{e}}$ siècles. Elle est l'auteur d'un ouvrage sur Arthur Conan Doyle (paru aux PU de Rennes en 2004) et, outre des articles consacrés à la fiction de la seconde moitié du XIX ${ }^{\mathrm{e}}$ siècle, elle a par ailleurs publié plusieurs articles sur la littérature britannique contemporaine (David Mitchell, Patrick McGrath, K. Ishiguro, K. MacLeod, W. Self, J. Winterson) et anglophone (D. Meyer). Elle travaille également sur les séries TV, plus particulièrement les fictions policières, fantastiques et de SF. Elle s'est spécialisée dans la résurgence des figures mythiques dans la littérature et les arts contemporains et a dirigé un ouvrage intitulé Le Savant fou, paru aux Presses Universitaires de Rennes en 2013. Suite aux projets InterMSH qu'elle a porté sur le posthumain, elle a publié deux ouvrages en collaboration : PostHumain(s), frontières, évolutions, hybridités est paru aux PU de Rennes en 2014 et le numéro 37 de la revue Otrante : « Mutations I : corps posthumains » chez Kimé en 2015. Autres publications : J-F Chassay, H. Machinal \& M. MarracheGouraud, Signatures du monstre, PUR, 2017, et E. Després \& H. Machinal, L'imaginaire en séries I, Otrante 42, Paris, Kimé, 2017. Elle est impliquée dans plusieurs projets de recherche, un projet inter-régional avec GUEST (saison 2), le projet MESH « Machines » de Lille 3, et le colloque « Posthumains et Subjectivités numériques » à Cerisy en juin 2016, qui poursuivent les collaborations avec l'UQAM, Paris 8 et Rennes 2. Enfin, elle participe au projet « Formes de l'apocalypse » qui réunit l'UBO, Paris 8 et Montpellier 3. 